\title{
Microwave-Assisted Synthesis of Novel 5-Trichloromethyl-4,5-dihydro-1H-1-pyrazole Methyl Esters under Solvent Free Conditions
}

\author{
Marcos A. P. Martins, * Paulo Beck, Pablo Machado, Sergio Brondani, Sidnei Moura, Nilo Zanatta, \\ Helio G. Bonacorso and Alex F. C. Flores
}

Núcleo de Química de Heterociclos (NUQUIMHE), Departamento de Química, Universidade Federal de Santa Maria, 97105-900 Santa Maria-RS, Brazil

\begin{abstract}
A preparação, com bons rendimentos (70-98\%), de doze novos ésteres etílicos de 5-triclorometil-4,5-diidro-1H-1-pirazóis, usando-se ambientalmente boas técnicas induzidas por microondas, é descrita. Estes compostos foram sintetizados a partir da reação de ciclocondensação de 1,1,1-tricloro-4-alcoxi-3-alquen-2-onas $\left[\mathrm{CCl}_{3} \mathrm{C}(\mathrm{O}) \mathrm{C}\left(\mathrm{R}^{2}\right)=\mathrm{C}\left(\mathrm{R}^{1}\right) \mathrm{OR}\right.$, onde $\mathrm{R}, \mathrm{R}^{2}=\mathrm{H}$, alquila; $\mathrm{R}^{1}=\mathrm{H}$, alquila e arila] com metil carboxilato hidrazina. As vantagens obtidas pelo uso de irradiações de microondas na ausência de solventes, em relação ao método convencional foram demonstradas.
\end{abstract}

Twelve novel 5-trichloromethyl-4,5-dihydro-1H-1-pyrazole ethyl esters have been synthesized in good yields $(70-98 \%)$ by using environmentally benign microwave induced techniques. The compounds were synthesized from the cyclocondensation of 1,1,1-trichloro-4alkoxy-3-alken-2-ones $\left[\mathrm{CCl}_{3} \mathrm{C}(\mathrm{O}) \mathrm{C}\left(\mathrm{R}^{2}\right)=\mathrm{C}\left(\mathrm{R}^{1}\right) \mathrm{OR}\right.$, where $\mathrm{R}, \mathrm{R}^{2}=\mathrm{H}$, alkyl; $\mathrm{R}^{1}=\mathrm{H}$, alkyl and aryl] with hydrazine methyl carboxylate. The advantages obtained by the using of microwave irradiation under solvent-free conditions, rather than a conventional method, were demonstrated.

Keywords: pyrazoles, enones, microwave irradiations, green chemistry

\section{Introduction}

Trihalomethyl substituted pyrazoles belong to an important class of compounds, which possess a wide variety of pharmaceutical and agrochemical properties. ${ }^{1,2}$ The main synthetic method used to prepare trihalomethylpyrazoles involves a [3+2] cyclization such as the classical 1,3-diketone with hydrazines. ${ }^{3}$ In recent years, we have developed a general synthesis of 1,1,1-trihalo-4-alkoxy-3-alken-2-ones, ${ }^{4,5}$ important halogen-containing building blocks and their use in heterocyclic preparations (e.g. isoxazoles, pyrazoles, pyrazolium chlorides, pyrrolidines, pyrimidines, thiazines, diazepines, thiazoles, selenazoles, and quinolines) has been extensively described. ${ }^{5}$ In particular, trichloromethyl substituted azoles are important as synthons and reagents in organic synthesis. These compounds have also been used as precursors for the synthesis of carbonyl-azole derivatives ${ }^{6}$ in a one-pot procedure. Moreover, the pharmaceutical properties of trichloromethyl substituted pyrazolines have been reported by our research group. ${ }^{7}$ Recently, we reported the application

* e-mail: mmartins@base.ufsm.br of microwave irradiation for the synthesis of halomethylsubstituted azoles. ${ }^{8}$ The beneficial effects of microwave irradiation are playing an increasing role in process chemistry, especially in cases where classical methods require forcing conditions or prolonged reaction times. When processes involve sensitive reagents, or there is the possibility of compound decomposition under prolonged reactions conditions, microwaves have also shown an advantage. The use of focused microwave irradiation to decrease reaction times and improve yields has been demonstrated. ${ }^{9}$ Microwave irradiation (MW), using commercial domestic ovens, has been recently used to accelerate organic reactions, due to its high heating efficiency, giving remarkable rate enhancement and dramatic reduction in reaction times. In recents papers were reported the application of microwave irradiation with great results for the synthesis of heterocycles, an eco-friendly methodology to prepare indazoles, pyrazolopyridines, bipyrazoles, aziridines, benzimidazoles and 2-oxazolines in solvent free conditions..$^{10,11}$ Thus, the aim of this work is to demonstrate the advantages obtained by the use of microwave irradiation for the synthesis of novel 5trichloromethyl-4,5-dihydro-1H-1-pyrazole methyl esters 
2 by the reaction of 1,1,1-trichloro-4-alkoxy-3-alken-2ones 1 with methyl hydrazino carboxylate under solvent free conditions and in good yields (Scheme 1).

The 1,1,1-trichloro-4-alkoxy-3-alken-2-ones 1a-l were synthesized from the reaction of the respective enol ether or acetal with trichloroacethyl chloride. ${ }^{4}$

Treatment of 1,1,1-trichloro-4-alkoxy-3-alken-2-ones 1 with methyl hydrazine carboxylate for six minutes, using microwaves $(45 \mathrm{~W})$ at $50-55{ }^{\circ} \mathrm{C}$, produced the 5-trichloromethyl-4,5-dihydro- $1 H$-1-pyrazole methyl esters 2 , under solvent free conditions. Conventional heating gave only moderate yields of the expected products, whereas with the use of microwave irradiation under solvent free conditions, the cyclocondensation products were obtained with a drastic reduction in reaction times (Table 1).

\section{Experimental}

Unless otherwise indicated, all common reagents and solvents were used as obtained from commercial supplies without further purifications. The melting points were taken on a melting point microscope Reichert-Thermovar and are uncorrected. ${ }^{1} \mathrm{H}$ and ${ }^{13} \mathrm{C}$ NMR spectra were recorded on a Bruker DPX $400\left({ }^{1} \mathrm{H}\right.$ at $400.13 \mathrm{MHz}$ and ${ }^{13} \mathrm{C}$ at $100.62 \mathrm{MHz}$ ) in $5 \mathrm{~mm}$ sample tubes at $298 \mathrm{~K}$ (digital resolution $\pm 0.01 \mathrm{ppm}$ ) in $\mathrm{CDCl}_{3} / \mathrm{TMS}$ solutions. Mass spectra were registered in a HP $6890 \mathrm{GC}$ connected to a HP 5973 MSD and interfaced by a Pentium PC. The GC was equipped with a split-splitless injector, autosampler, cross-linked HP-5 capillary column $(30 \mathrm{~m}, 0.32 \mathrm{~mm}$ of internal diameter), and helium was used as the carrier gas.

Microwave irradiations were conducted in a Panasonic M720 at a frequency of $2450 \mathrm{MHz}$, with an average energy in the sample of $45 \mathrm{~W}$ and the temperature measured after the completion of the reaction in the range of $50-55^{\circ} \mathrm{C}$. The measure of average energy in the sample is in agreement with methodology presented in reference 9.

Synthesis of 5-trichloromethyl-4,5-dihydro-1H-1-pyrazole ethyl esters $\mathbf{2 a}-\mathbf{l}$ (microwave method)

A mixture of 1,1,1-trichloro-4-alkoxy-3-alken-2-ones 1 ( $2 \mathrm{mmol}$ ) and methyl hydrazino carboxylate $(2.5 \mathrm{mmol})$ was stirred for a few minutes, and then the mixture was irradiated in a microwave oven at $45 \mathrm{~W}$ for $6 \mathrm{~min}$, at $50-55{ }^{\circ} \mathrm{C}$ (temperature after the completion of the reaction). A10\% $\mathrm{HCl}$ solution $(30 \mathrm{~mL})$ was added to the reaction mixture, and the product 2 was extracted with chloroform $(2 \times 20$ $\mathrm{mL})$, washed with distilled water $(2 \times 30 \mathrm{~mL})$ and dried with $\mathrm{MgSO}_{4}$. The solvent was removed in a rotaevaporator, and the product was obtained in high purity. When necessary the product was recrystallized from cyclohexane.

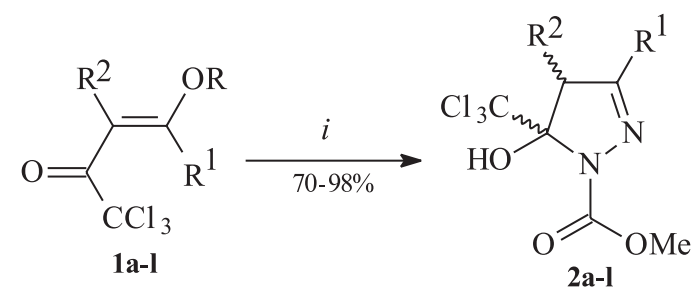

${ }^{i}$ Reaction Conditions: $\mathrm{NH}_{2} \mathrm{NHC}(\mathrm{O}) \mathrm{OMe}, 45 \mathrm{~W}, 50-55{ }^{\circ} \mathrm{C}, 6 \mathrm{~min}$.

\begin{tabular}{ccccccccccccc}
\hline $\mathbf{1 , 2}$ & $\mathbf{a}$ & $\mathbf{b}$ & $\mathbf{c}$ & $\mathbf{d}$ & $\mathbf{e}$ & $\mathbf{f}$ & $\mathbf{g}$ & $\mathbf{h}$ & $\mathbf{i}$ & $\mathbf{j}$ & $\mathbf{k}$ & $\mathbf{l}$ \\
\hline $\mathrm{R}^{1}$ & $\mathrm{H}$ & $\mathrm{Me}$ & $\mathrm{Et}$ & ${ }^{n} \mathrm{Pr}$ & ${ }^{i} \mathrm{Pr}$ & ${ }^{c y c l o} \mathrm{Pr}$ & ${ }^{n} \mathrm{Bu}$ & ${ }^{i} \mathrm{Bu}$ & ${ }^{t} \mathrm{Bu}$ & $\mathrm{H}$ & $\mathrm{Ph}$ & $4-\mathrm{O}_{2} \mathrm{~N}_{-} \mathrm{C}_{6} \mathrm{H}_{4}$ \\
$\mathrm{R}^{2}$ & $\mathrm{H}$ & $\mathrm{H}$ & $\mathrm{H}$ & $\mathrm{H}$ & $\mathrm{H}$ & $\mathrm{H}$ & $\mathrm{H}$ & $\mathrm{H}$ & $\mathrm{H}$ & $\mathrm{Me}$ & $\mathrm{H}$ & $\mathrm{H}$ \\
$\mathrm{R}$ & $\mathrm{Et}$ & $\mathrm{Me}$ & $\mathrm{Me}$ & $\mathrm{Me}$ & $\mathrm{Me}$ & $\mathrm{Me}$ & $\mathrm{Me}$ & $\mathrm{Me}$ & $\mathrm{Me}$ & $\mathrm{Et}$ & $\mathrm{Me}$ & $\mathrm{Me}$ \\
\hline
\end{tabular}

Scheme 1.

Table 1. Yields ${ }^{\mathrm{a}}$ and reaction conditions $\mathrm{s}^{\mathrm{b}}$ used for the microwave assisted synthesis of $\mathbf{2}$

\begin{tabular}{|c|c|c|c|c|c|c|c|c|c|}
\hline \multirow[b]{2}{*}{ Product } & \multicolumn{2}{|c|}{ Microwave method ${ }^{\mathrm{b}}$} & \multicolumn{2}{|c|}{ Conventional method } & \multirow[b]{2}{*}{ Product } & \multicolumn{2}{|c|}{ Microwave method ${ }^{b}$} & \multicolumn{2}{|c|}{ Conventional method } \\
\hline & Reaction time ( $\mathrm{min}$ ) & Yield (\%) & Reaction time (h) & Yield(\%) & & Reaction time (min) & Yield (\%) & Reaction time (h) & Yield (\%) \\
\hline $2 a$ & 6 & 71 & 24 & 70 & $2 \mathrm{~g}$ & 6 & 86 & 24 & 79 \\
\hline $2 \mathbf{b}$ & 6 & 70 & 24 & 72 & $2 \mathrm{~h}$ & 6 & 89 & 24 & 70 \\
\hline $2 c$ & 6 & 98 & 24 & 78 & $2 \mathbf{i}$ & 6 & 87 & 24 & 75 \\
\hline 2d & 6 & 92 & 24 & 75 & $2 \mathbf{j}$ & 6 & 80 & 24 & 73 \\
\hline $2 \mathrm{e}$ & 6 & 76 & 24 & 70 & $2 k$ & 6 & 75 & 24 & 74 \\
\hline $2 f$ & 6 & 93 & 24 & 78 & 21 & 6 & 70 & 24 & 70 \\
\hline
\end{tabular}

${ }^{\mathrm{a}}$ Yields of isolated products; ${ }^{\mathrm{b}}$ reaction conditions: solvent free, $\mathrm{MW}, 45 \mathrm{~W}, 50-55^{\circ} \mathrm{C}$. 
Synthesis of 5-trichloromethyl-4,5-dihydro-1H-1-pyrazole ethyl esters $2 \boldsymbol{a}-\boldsymbol{l}$ (conventional method)

A solution of 1 (2 mmol) and $\mathrm{NH}_{2} \mathrm{NHCO}_{2} \mathrm{Me}(2.5$ $\mathrm{mmol})$ in methanol $(30 \mathrm{~mL})$ was stirred under reflux for 24 hours. Water was added $(60 \mathrm{~mL})$ and the organic phase was extracted with $\mathrm{CHCl}_{3}(2 \times 20 \mathrm{~mL})$. The organic extract was dried $\left(\mathrm{MgSO}_{4}\right)$ and the solvent was removed under reduced pressure. The work-up was carried out as described for the microwave method.

2a. $\mathrm{C}_{6} \mathrm{H}_{7} \mathrm{Cl}_{3} \mathrm{~N}_{2} \mathrm{O}_{3}$, mw 261.48, oil. ${ }^{1} \mathrm{H}$ NMR $\delta(J, \mathrm{~Hz})$ 7.10 (s, 1H, H-3), 3.28 (d, 1H, J 19, H-4a), 3.71 (d, 1H, J 19, H-4b), 3.90 (s, 3H, OMe). ${ }^{13} \mathrm{C}$ NMR $\delta 154.82(\mathrm{C}=\mathrm{O})$, 146.6 (C-3), 103.2 (C-5), $98.8\left(\mathrm{CCl}_{3}\right), 53.6$ (OMe), 47.2 (C-4). Anal. Calc.: C, 27.56\%; H, 2.70\%; N, $10.71 \%$. Found: C, 27.43\%; H, 2.69\%; N, 10.66\%. MS m/z, (\%) $143\left(\mathrm{M}^{+}-\mathrm{CCl}_{3}, 100\right) ; 111$ (52), 59 ( $\left.\mathrm{CO}_{2} \mathrm{Me}, 26\right)$.

2b. $\mathrm{C}_{7} \mathrm{H}_{9} \mathrm{Cl}_{3} \mathrm{~N}_{2} \mathrm{O}_{3}$, mw 275.51 , mp $101-103{ }^{\circ} \mathrm{C} .{ }^{1} \mathrm{H}$ NMR $\delta(J, \mathrm{~Hz}) 3.29$ (d, 1H, $J$ 19, H-4a) 3.57 (d, 1H, $J$ 19, H4b), 2.09 (s, 3H, Me), 3.89 (s, 3H, OMe). ${ }^{13} \mathrm{C}$ NMR $d 156.7$ (C-3), 154.0 (C=O ), 111.1 (C-5), $101.9\left(\mathrm{CCl}_{3}\right), 53.4(\mathrm{OMe})$, 47.9 (C-4), 15.5 (Me). Anal. Calc.: C, 30.52\%; H, 3.29\%; N, $10.17 \%$. Found: C, 30.48\%; H, 3.28\%; N, 10.15\%. MS m/z, (\%) $157\left(\mathrm{M}^{+}-\mathrm{CCl}_{3}, 99\right) 125$ (100), 83 (38).

2c. $\mathrm{C}_{8} \mathrm{H}_{11} \mathrm{Cl}_{3} \mathrm{~N}_{2} \mathrm{O}_{3}$, mw 289.53, mp $76-78{ }^{\circ} \mathrm{C} .{ }^{1} \mathrm{H}$ NMR $\delta(J, \mathrm{~Hz}) 3.71$ (d, 1H, $J$ 19, H-4a), 3.24 (d, 1H, $J$ 19, H-4b) 3.90 (s, 3H, OMe), 3.26 (q, 2H, $\mathrm{CH}_{2}$ ), 2.01 (t, $\left.3 \mathrm{H}, \mathrm{CH}_{3}\right) .{ }^{13} \mathrm{C}$ NMR $\delta 161.7(\mathrm{C}-3), 154.7(\mathrm{C}=\mathrm{O}), 111.7$ (C-5), $102.7\left(\mathrm{CCl}_{3}\right), 53.3(\mathrm{OMe}), 47.1(\mathrm{C}-4) 23.0\left(\mathrm{CH}_{2}\right)$ $10.0\left(\mathrm{CH}_{3}\right)$. Anal. Calc.: C, 33.19\%; H, 3.83\%; N, $9.67 \%$. Found: C, 33.20\%; H, 3.80\%, N, 9.66\%. MS m/z, (\%) $171\left(\mathrm{M}^{+}-\mathrm{CCl}_{3}, 100\right), 139(81), 111(47)$.

2d. $\mathrm{C}_{9} \mathrm{H}_{13} \mathrm{Cl}_{3} \mathrm{~N}_{2} \mathrm{O}_{3}$, mw 303.56, mp 59-61 ${ }^{\circ} \mathrm{C} .{ }^{1} \mathrm{H}$ NMR $\delta(J, \mathrm{~Hz}) 3.71$ (d, 1H, J 19, H-4a), 3.24 (d, 1H, J 19, H4b) $3.81(\mathrm{~s}, 3 \mathrm{H}, \mathrm{OMe}) 2.29\left(\mathrm{t}, 2 \mathrm{H}, \mathrm{CH}_{2}\right), 1.57(\mathrm{~m}, 2 \mathrm{H}$, $\left.\mathrm{CH}_{2}\right), 0.91\left(\mathrm{t}, 3 \mathrm{H}, \mathrm{CH}_{3}\right),{ }^{13} \mathrm{C} \mathrm{NMR} \delta 164.7$ (C-3), 160.0 $(\mathrm{C}=\mathrm{O}), 108.0(\mathrm{C}-5), 105.0\left(\mathrm{CCl}_{3}\right) 53.4(\mathrm{OMe}), 53.0(\mathrm{C}-4)$ 36.8, $24.7\left(2 \mathrm{CH}_{2}\right) 18.7\left(\mathrm{CH}_{3}\right)$. Anal. Calc.: C, 35.61\%; $\mathrm{H}$, $4.32 \%$; N, 9.23\%. Found: C, 35.37\%; H, 4.29\%, N, 9.17\%. MS m/z, (\%) 185 (100), 139 (76), 111 (42).

2e. $\mathrm{C}_{9} \mathrm{H}_{13} \mathrm{Cl}_{3} \mathrm{~N}_{2} \mathrm{O}_{3}$, mw 303.56, mp $63-65^{\circ} \mathrm{C}$. ${ }^{1} \mathrm{H}$ NMR $\delta(J, \mathrm{~Hz}) 3.50$ (d, 1H, J 19, H-4a), 3.28 (d, 1H, J 19, H4b) 3.88 (s, 3H, OMe), $2.79(\mathrm{~m}, 1 \mathrm{H}, \mathrm{CH}), 1.21(\mathrm{~d}, 3 \mathrm{H}$, $\left.\mathrm{CH}_{3}\right), 1.18\left(\mathrm{~d}, 3 \mathrm{H}, \mathrm{CH}_{3}\right) .{ }^{13} \mathrm{C}$ NMR $\delta 164.1$ (C-3), 155.0 $(\mathrm{C}=\mathrm{O}), 111.6(\mathrm{C}-5), 102.8\left(\mathrm{CCl}_{3}\right), 53.6(\mathrm{OMe}), 45.3(\mathrm{C}-$ 4), $25.3(\mathrm{CH}), 22.0\left(2 \mathrm{CH}_{3}\right)$. Anal. Calc.: C, 35.61\%; H,
$4.32 \%$; N, 9.23\%. Found: C, 35.60\%; H, 4.22\%, N, 9.24\%. $\mathrm{MS} m / z,(\%) 185\left(\mathrm{M}^{+}-\mathrm{CCl}_{3}, 100\right), 153$ (47), 111 (71).

2f. $\mathrm{C}_{9} \mathrm{H}_{11} \mathrm{Cl}_{3} \mathrm{~N}_{2} \mathrm{O}_{3}$, mw 301.55, mp $113-115{ }^{\circ} \mathrm{C} .{ }^{1} \mathrm{H}$ NMR $\delta(J, \mathrm{~Hz}) 3.30$ (d, 1H, $J$ 19, H-4a), 3.15 (d, 1H, $J$ 19, H-4b) 3.88 (s, 3H, OMe), $1.30(\mathrm{CH}), 0.95,0.85$ (m, $\left.4 \mathrm{H}, 2 \mathrm{CH}_{2}\right) .{ }^{13} \mathrm{C}$ NMR $\delta 161.5(\mathrm{C}-3), 155.0(\mathrm{C}=\mathrm{O}), 103.5$ (C-5), $99.9\left(\mathrm{CCl}_{3}\right), 53.7(\mathrm{OMe}), 45.7(\mathrm{C}-4), 11.3(\mathrm{CH})$, 6.68, $6.51\left(2 \mathrm{CH}_{2}\right)$. Anal. Calc.: C, 35.85\%; H, 3.68\%; N, $9.29 \%$. Found: C, $35.84 \%$; H, 3.65\%, N, 9.21\%. MS $\mathrm{m} / \mathrm{z}$, (\%) $183\left(\mathrm{M}^{+}-\mathrm{CCl}_{3}, 100\right), 151$ (55).

2g. $\mathrm{C}_{10} \mathrm{H}_{15} \mathrm{Cl}_{3} \mathrm{~N}_{2} \mathrm{O}_{3}$, mw 317.59, mp $59-61{ }^{\circ} \mathrm{C} .{ }^{1} \mathrm{H}$ NMR $\delta(J, H z) 3.50$ (d, 1H, $J$ 19, H-4a), 3.23 (d, 1H, $J$ 19, H-4b) 3.86 (s, 3H, OMe), 2.38 (m, 2H, $\mathrm{CH}_{2}$ ), 1.53 (m, $\left.2 \mathrm{H}, \mathrm{CH}_{2}\right), 1.36\left(\mathrm{~m}, 2 \mathrm{H}, \mathrm{CH}_{2}\right), 0.91\left(\mathrm{t}, 3 \mathrm{H}, \mathrm{CH}_{3}\right) .{ }^{13} \mathrm{C} \mathrm{NMR}$ $\delta 159.7(\mathrm{C}-3), 154.7(\mathrm{C}=\mathrm{O}), 111.6(\mathrm{C}-5), 102.7\left(\mathrm{CCl}_{3}\right)$, 47.2 (C-4), 53.2 (OMe), 30.9, 29.6, $22.0\left(3 \mathrm{CH}_{2}\right), 13.55$ $\left(\mathrm{CH}_{3}\right)$. Anal. Calc.: C, 37.82\%; H, 4.76\%; N, 8.82\%. Found: C, 37.57\%; H, 4.73\%, N, 8.76\%. MS m/z, (\%) $199\left(\mathrm{M}^{+}-\mathrm{CCl}_{3}, 100\right), 167$ (32), 111 (14), 57 (73).

2h. $\mathrm{C}_{10} \mathrm{H}_{15} \mathrm{C}_{13} \mathrm{~N}_{2} \mathrm{O}_{3}$, mw 317.59, mp $57-59{ }^{\circ} \mathrm{C} .{ }^{1} \mathrm{H}$ NMR $\delta(J, H z) 3.55$ (d, 1H, $J$ 19, H-4a), 3.22 (d, 1H, $J$ 19, H-4b) 3.89 (s, 3H, OMe), 2.29 (d, 2H, $\mathrm{CH}_{2}$ ), 1.95 (m, $1 \mathrm{H}, \mathrm{CH}), 1.0\left(\mathrm{~d}, 6 \mathrm{H}, 2 \mathrm{CH}_{3}\right) .{ }^{13} \mathrm{C}$ NMR $\delta 159.2(\mathrm{C}-3), 155.1$ $(\mathrm{C}=\mathrm{O}), 111.6$ (C-5), $102.8\left(\mathrm{CCl}_{3}\right), 47.2$ (C-4), 53.6 (OMe), $38.9\left(\mathrm{CH}_{2}\right), 26.2(\mathrm{CH}), 22.7,22.3\left(2 \mathrm{CH}_{3}\right)$. Anal. Calc.: C, $37.82 \%$; H, 4.76\%; N, 8.82\%. Found: C, 37.49\%; H, $4.72 \%, \mathrm{~N}, 8.73 \%$. MS m/z, (\%) $199\left(\mathrm{M}^{+}-\mathrm{CCl}_{3}, 100\right), 167$ (24), 57 (90).

2i. $\mathrm{C}_{10} \mathrm{H}_{15} \mathrm{Cl}_{3} \mathrm{~N}_{2} \mathrm{O}_{3}$, mw 317.59, mp $105-107{ }^{\circ} \mathrm{C} .{ }^{1} \mathrm{H}$ NMR $\delta(J, H z) 3.59$ (d, 1H, J 19, H-4a), 3.26 (d, 1H, $J$ 19, H-4b), 3.87 (s, 3H, OMe), 1.23 (s, 9H, 3CH $).{ }^{13} \mathrm{C}$ NMR $\delta 165.6(\mathrm{C}-3), 154.7(\mathrm{C}=\mathrm{O}), 103.2(\mathrm{C}-5), 99.7\left(\mathrm{CCl}_{3}\right)$, $52.9(\mathrm{OMe}), 44.8(\mathrm{C}-4), 33.6\left(\mathrm{C}-{ }^{t} \mathrm{Bu}\right), 27.0\left(3 \mathrm{CH}_{3}\right)$. Anal. Calc.: C, $37.82 \%$; H, 4.76\% ; , 8.82\%. Found: C, 37.45\%; $\mathrm{H}, 4.71 \%$; N, 8.72\%. MS m/z, (\%) $199\left(\mathrm{M}^{+}-\mathrm{CCl}_{3}, 99\right)$, 167 (14), 57(100).

2j. $\mathrm{C}_{7} \mathrm{H}_{9} \mathrm{Cl}_{3} \mathrm{~N}_{2} \mathrm{O}_{3}$, mw 275.51, mp $95-97^{\circ} \mathrm{C} .{ }^{1} \mathrm{H}$ NMR $\delta 7.0$ (s, 1H, H-3), 3.90 (s, 3H, OMe), 3.64 (q, 1H, H-4a), $1.27\left(\mathrm{~d}, 3 \mathrm{H}, \mathrm{CH}_{3}\right) .{ }^{13} \mathrm{C}$ NMR $\delta 155.4(\mathrm{C}-3), 151.7(\mathrm{C}=\mathrm{O})$, 104.3 (C-5), $98.7\left(\mathrm{CCl}_{3}\right), 53.8$ (OMe), 50.4 (C-4) 12.1 $\left(\mathrm{CH}_{3}\right)$. Anal. Calc.: C, 30.52\%; H, 3.29\%; N,10.17\%. Found:C, 30.24\%; H, 3.26\%; N, 10.07\%. MS m/z, (\%) $157\left(\mathrm{M}^{+}-\mathrm{CCl}_{3}, 100\right), 125$ (77), 97 (43).

2k. $\mathrm{C}_{12} \mathrm{H}_{11} \mathrm{Cl}_{3} \mathrm{~N}_{2} \mathrm{O}_{3}$, mw 337.58, mp $141-143{ }^{\circ} \mathrm{C}$. ${ }^{1} \mathrm{H}$ NMR $\delta(J, \mathrm{~Hz}) 3.76$ (d, 1H, $J$ 19, H-4a), 3.50 (d, 1H, $J$ 
19, H-4b), 3.95 (s, 3H, OMe), 7.41-7.74 (m, 5H, Ph). ${ }^{13} \mathrm{C}$ NMR $\delta 154.0(\mathrm{C}-3), 153(\mathrm{C}=\mathrm{O}), 131.0-121.0(6 \mathrm{C}, \mathrm{Ph})$, 112.8 (C-5), $90.0\left(\mathrm{CCl}_{3}\right), 54.0$ (OMe), 43.2 (C-4). Anal. Calc.: C, $42.70 \%$; H, 3.28\%; N, 8.30\%. Found:C, 42.57\%; $\mathrm{H}, 3.26 \%$;, $8.26 \%$. MS m/z, (\%) $219\left(\mathrm{M}^{+}-\mathrm{CCl}_{3}, 100\right)$, 77 (Ph, 80), 187 (30), 288 (82).

2l. $\mathrm{C}_{12} \mathrm{H}_{10} \mathrm{Cl}_{3} \mathrm{~N}_{3} \mathrm{O}_{5}$, mw 382.57, mp $158-160{ }^{\circ} \mathrm{C} .{ }^{1} \mathrm{H}$ NMR $\delta(J, \mathrm{~Hz}) 4.04(\mathrm{~d}, 1 \mathrm{H}, J$ 19, H-4a), $3.83(\mathrm{~d}, 1 \mathrm{H}, J$ 19, H-4b), 3.96 (s, 3H, OMe), 7.91 (d, 2H, Ph), 8.29 (d, $2 \mathrm{H}, \mathrm{Ph}) .{ }^{13} \mathrm{C}$ NMR $\delta 155.0(\mathrm{C}-3), 152.6(\mathrm{C}=\mathrm{O}), 103.1(\mathrm{C}-$ 5), $101.1\left(\mathrm{CCl}_{3}\right), 54.0$ (OMe), 46.0 (C-4), 147.0 - 123.0 (6C, Ph). Anal. Calc.: C, 37.68\%; H, 2.63\%; N,10.98\%. Found: C, 37.60\%; H, 2.61\%; N, 10.92\%.MS $\mathrm{m} / \mathrm{z}$, (\%) $263\left(\mathrm{M}+1-\mathrm{CCl}_{3}, 90\right), 218(100), 59(60)$.

\section{Acknowledgments}

The authors thank the Conselho Nacional de Desenvolvimento Científico e Tecnológico (CNPq/ PADCT) and Fundação de Amparo à pesquisa do Estado do Rio Grande do Sul (FAPERGS) for financial support. The fellowships from CNPq, CAPES and FAPERGS are also acknowledged.

\section{References}

1. Hainzl, D.; Cole, L.M.; Casida, J.E.; Chem. Res. Toxicol. 1998, 11, 1529.

2. Talley, J. J.; Bronw, D. L.; Carter, J. S.; Graneto, M. J.; Kobolt, C. M.; Mansferrer, J. L.; Perkins, W. E.; Rogers, R. S.; Shaffer, A. F.; Zhang, Y. Y.; Zweifel, B. S.; Seibert, K.; J. Med. Chem. 2000, 43, 775; J. Med. Chem. 1997, 40, 1347; Djuric, S. W.; BaMaung, N. Y.; Basha, A.; Liu, H.; Luly, J. R.; Madar, D. J.; Sciotti, R. J.; Tu, N. P.; Wagenaar, F. L.; Wiedman, P. E.; Zhou, X.; Ballaron, S.; Bauch, J.; Chen, Y.- W.; Chiou, X. G.; Fey, T.; Gauvin, D.; Gubbins, E.; Hsieh, G. C.; Marsch, K. C.; Mollison, K. W.; Pong, M.; Shaughnessy, T. K. Sheets, M. P.; Smith, M.; Trevillyan, J. M.; Warrior, U.; Wegner, C. D.; Carter, G. W.; J. Med. Chem. 2000, 43, 2975.

3. Pushkevich, K. I.; Saloutin, V. I.; Postovskii, I.; Russ. Chem. Rev. 1981, 50, 180; Lyga, J. W.; Patera, R. M.; J. Heterocycl. Chem. 1990, 27, 919; Claire, P. P. K.; Coe, P. L.; Jones, C. J.; McCleverty, J. A.; J. Fluorine Chem. 1991, 51, 283; Bumgardner, C. L.; Sloop, J. C.; J. Fluorine Chem. 1992, 56, 141; Song, L.; Chu, Q.; Zhu, S.; J. Fluorine Chem. 2001, 107, 1076; Song, L.; Chu, Q.; Zhu, S.; J. Fluorine Chem. 2001, 111, 201; Singh, S. P.; Kumar, D.; Jones, B. G.; Threadgill, M. D.; J. Fluorine Chem. 1999, 94, 199.
4. Colla, A.; Martins, M. A. P.; Clar, G.; Krimmer, S.; Fischer, P.; Synthesis 1991, 483; Martins, M. A. P.; Bastos, G. P.; Bonacorso, H. G.; Zanatta, N.; Flores, A. F. C.; Siqueira, G. M.; Tetrahedron Lett. 1999, 40, 4309; Bonacorso, H. G.; Martins, M. A. P.; Bittencourt, S. R. T.; Lourega, R. V.; Zanatta, N.; Flores, A. F. C.; J. Fluorine Chem. 1999, 99, 177; Martins, M.A.P.; Emmerich, D.J.; Pereira, C.M.P.; Cunico, W.; Rossato, M.; Zanatta, N.; Bonacorso, H.G.; Tetrahedron Lett. 2004, 45, 4935.

5. Martins, M. A. P.; Cunico, W.; Pereira, C.M.P; Flores, A.F.C.; Bonacorso H.G.; Zanatta, N.; Curr. Org. Synth. 2004, 1, 391.

6. Martins, M. A. P.; Freitag, R.; Flores, A.F.C.; Zanatta, N.; Synthesis 1995, 1491; Martins, M. A. P.; Flores, A.F.C.; Bastos, G. P.; Sinhorin, A.; Bonacorso, H.G.; Zanatta, N.; Tetrahedron Lett. 2000, 41, 293; Flores, A. F. C.; Zanatta, N.; Rosa, A.; Brondani, S.; Martins, M. A. P.; Tetrahedron Lett. 2002, 43, 5005; Martins, M. A. P.; Emmerich, D.; Beck, P.; Cunico, W.; Pereira, C.M.P.; Sinhorin, A.P.; Brondani, S.; Peres, R.; Teixeira, M.V.M.; Bonacorso, H.G.; Zanatta, N.; Synth. Commun. 2004, 10, 1915.

7. Souza, F. R.; Souza, V. T.; Ratzlaff, V.; Borges, L. P.; Oliveira, M. R.; Bonacorso, H. G.; Zanatta, N.; Martins, M. A. P.; Mello, C. F. Eur. J. Pharmacol. 2002, 451, 141; Godoy, M. C. M.; Fighera, M. R.; Souza, F. R.; Flores, A. E.; Rubin, M. A.; Oliveira, M. R.; Zanatta, N.; Martins, M. A. P.; Bonacorso, H. G.; Mello, C. F.; Eur. J. Pharmacol. 2004, 496, 93; Tabarelli, Z.; Rubin, M. A.; Berlese, D. B.; Sauzem, P. D.; Missio, T. P.; Teixeira, M. V.; Sinhorin, A. P.; Martins, M. A. P. ; Zanatta, N.; Bonacorso, H. G.; Mello, C. F.; Brazilian J. Med. Biol. Res. 2005, 37, 1531.

8. Martins, M. A. P.; Beck, P.; Cunico, W.; Pereira, C. M. P.; Sinhorin, A. P.; Blanco, R. F.; Peres, R.; Bonacorso, H. G.; Zanatta, N.; Tetrahedron Lett. 2002, 43, 7005; Martins, M.A.P.; Blanco, R.F.; Pereira, C.M.P; Beck, P.; Brondani, S.; Cunico, W.; Bonacorso, H.G.; Zanatta, N.; Tetrahedron Lett. 2003, 44, 6669.

9. Kingston, H. M.; Jassie, L. B.; Introduction to Microwave Sample Preparation - Theory and Practice, ACS: Washington, 1988; Pecoraro, E.; Davolos, M. R.; Jafelicci, M. J.; Quim. Nova 1997, 20, 89; Lidström, P.; Tierney, J.; Wathey, B.; Westman, J.; Tetrahedron 2001, 57, 9225.

10. Díaz-Ortiz, A.; la Hoz, A.; Langa, F.; Green Chem. 2000, 2 , 165.

11. Varma, R. S.; Green Chem. 1999, 1, 43; Varma, R. S.; Pure Appl. Chem. 2001, 1, 193.

Received: September 21, 2005 Published on the web: February 13, 2006 